\title{
CENÁRIO DAS INTERNAÇÕES PSIQUIÁTRICAS COMPULSÓRIAS EM UM MUNICÍPIO DO SUL DO BRASIL
}

Robsmeire Calvo Melo Zurita ${ }^{1}$, Rebeca Melo Zurita ${ }^{2}$, Rosana Rosseto de Oliveira ${ }^{1, E m i l i a n a}$ Cristina Melo', Julyanna Bassan Ripke ${ }^{3}$

${ }^{1}$ Enfermeira. Doutoranda da Pós Graduação de Enfermagem da Universidade Estadual de Maringá (UEM). ${ }^{2}$ Acadêmica de Medicina. Universidade Federal do Mato Grosso (UFMT). ${ }^{3}$ Enfermeira. Egressa do Centro Universitário CESUMAR (UNICESUMAR). E-mail: robszurita@bol.com.br

\section{RESUMO}

As internações psiquiátricas são classificadas em voluntárias quando acontecem com consentimento da pessoa internada; involuntárias que ocorrem sem o consentimento do usuário e a pedido de terceiro; e compulsórias que são determinadas pela justiça. Este estudo teve por objetivo analisar as internações psiquiátricas compulsórias no município de Maringá-Paraná, de 2009 a 2011 na Emergência Psiquiátrica (EP) do Hospital Municipal de Maringá Thelma Villanova Kasprowicz. Foram utilizados dados secundários do fluxo diário da EP. Foram pesquisadas as seguintes variáveis: internação compulsória por tipo de transtorno mental ou dependência química, idade e sexo. Encontrou-se aumento gradativo das internações psiquiátricas compulsórias, sendo na maioria pessoas do sexo masculino; de 16 a 20 anos de idade e internações por drogas. A droga é problema de saúde coletiva onde políticas públicas podem ser transformadas em ações efetivas e resolutivas, com serviços substitutivos e profissionais habilitados para atuarem com este público alvo.

PALAVRAS-CHAVE: Enfermagem, internação compulsória de doente mental, saúde mental.

\section{INTRODUÇÃO}

Quanto à regulamentação, as internações são classificadas em voluntárias quando acontecem com o consentimento da pessoa que vai ser internada; involuntárias que ocorrem sem o consentimento do usuário e a pedido de terceiro e as compulsórias que são determinadas pela justiça (BRITTO, 2004).

A Internação Psiquiátrica Compulsória prevista na Lei 10.216 de 2001 é aquela efetuada por determinação da autoridade judicial, quando a pessoa com transtorno mental oferece risco para si, para sua família ou para a sociedade, levando-se em conta as condições de segurança do estabelecimento, quanto à salvaguarda do paciente, dos demais internados e funcionários. Por ser uma medida judicial, não podem ser legalmente questionadas, embora sobre a ótica ético-moral, mereçam discussões profundas (FORTES, 2010).

Poucos estudos trazem um panorama das doenças de notificação compulsória no Brasil. Mendes et al (2000) encontraram que em 1993, houve 204.842 internações por notificação compulsória, representando 1,38\% do total das internações realizadas. Já em 1998, registraram-se 
116.700 internações por doenças de notificação compulsória, correspondendo a 1\% do total, com redução em termos absolutos de 88.142 internações realizadas pelo Sistema Único de Saúde no país nesse período.

No Brasil, a partir da segunda metade do século XIX, ainda durante o período imperial, teve início a assistência psiquiátrica pública, que era exercida de forma leiga mediante instituições de caráter asilar pertencentes à Igreja Católica e instituições de caridade. Com a alteração da situação social e econômica que havia determinado o nascimento do hospício, o emergente capitalismo pedia novas providências. O processo político em curso, advindo da proclamação da República, resultou em novos confrontos de poder entre o Estado emergente, a classe médica, e a classe clerical. Este período foi configurado como o marco divisório entre a psiquiatria empírica e a psiquiatria científica. Até o final da década de 1950, tratar o doente mental continuava essencialmente restrito ao interior dos hospitais (FORTES, 2010).

No final da década de 80 e início de 90 surgiram experiências de transformação da assistência psiquiátrica para a Rede de Atenção Integral em Saúde Mental, cuja ampla estrutura destina-se ao atendimento de necessidades diversas, abrange serviços de todos os níveis de atenção, desde a atenção básica até os serviços especializados como centros de atenção psicossocial, serviços residenciais terapêuticos, ambulatórios de saúde mental, hospitais dia, leitos/unidades em hospitais gerais, serviços de urgência/emergência psiquiátrica, geração de renda e trabalho, centros de convivência, unidades de acolhimento e consultório na rua (MIELKE et al, 2011).

As mudanças geradas no modelo de assistência em saúde mental no Brasil e em muitos países no mundo apresentam crescente valorização de tratamentos de base comunitária, buscando além da remissão de sintomas, a reinserção social e a melhora das condições de vida das pessoas afetadas (NAGAOKA et al, 2011).

É inerente à profissão de enfermagem o cuidado prestado tanto ao indivíduo quanto a sua família, de maneira a garantir-lhes boas condições físicas e mentais, permitindo valorizar os mecanismos de enfrentamento das adversidades, da dor e do sofrimento. Para isso, a enfermagem deve incorporar novos conceitos, estar aberta a novos questionamentos, descobertas e propostas de ação (DIAS, 2010).

Ao observamos a realidade, vemos que a internação psiquiátrica ocorre com muita frequência de forma compulsória, não considerando dessa maneira que a pessoa deveria ter o direito de escolher o seu tratamento, sem ter a sua liberdade de ir e vir subtraída. Além disso, 
questiona-se o motivo para a ocorrência das internações compulsórias, uma vez que podem representar problemas de saúde pública.

Assim este estudo tem por objetivo analisar as internações psiquiátricas compulsórias no município de Maringá-Paraná de 2009 a 2011, a fim de identificar qual transtorno mental e/ou dependência química causam mais internações psiquiátricas compulsórias na Emergência Psiquiátrica do Hospital Municipal de Maringá Thelma Villanova Kasprowicz.

\section{METODOLOGIA}

Trata-se de um estudo descritivo, analítico, de natureza quantitativa. Foram utilizados dados secundários do fluxo diário da Emergência Psiquiátrica (EP) do Hospital Municipal de Maringá Thelma Villanova Kasprowicz. Foram pesquisadas as seguintes variáveis: internação compulsória por tipo de transtorno mental ou dependência química, idade e sexo, no município de Maringá no período de 2009 a 2011.

A EP do Hospital Municipal de Maringá Thelma Villanova Kasprowicz conta com 26 leitos psiquiátricos, sendo 14 leitos masculinos e 12 leitos femininos. Foi implantada em 2003, para atender a demanda das três Regionais de Saúde $11^{\circ}, 13^{\circ}$ e $15^{\circ}$ que inclui 67 municípios. $O$ paciente internado compulsoriamente na EP do Hospital Municipal de Maringá terá o término da sua internação involuntária quando houver solicitação por escrito do familiar, ou responsável legal, ou quando estabelecido pelo especialista responsável pelo tratamento, conforme artigo 8 da Lei № 10.216 de 2001.

A EP utiliza o software de Fluxo Diário para armazenar os dados de internações psiquiátricas, sendo organizados em planilhas por data de entrada na instituição. Neste software os principais dados são: nome, sexo, idade, município, regional de saúde, tipo droga, medicação, CID 10, diagnóstico, entrada, horário, saída, forma de procedência, encaminhamento, internação, dados clínicos, escolaridade, estado civil, filhos, religião, endereço.

Durante a coleta de dados nos registros do fluxo diário como a maioria das internações encontravam-se com mais de um CID 10, elegeu-se o principal para a coleta desse estudo.

O projeto aprovado pelo Centro de Capacitação Permanente em Saúde (CECAPS) ofício n. ${ }^{\circ} 477 / 2012$ da Secretaria Municipal de Saúde de Maringá-Paraná e pelo Comitê de Ética em Pesquisa do Cesumar (CEP), sob n. 46642 de 15/06/2012.

\section{RESULTADOS}


Os resultados encontrados das 103 internações compulsórias ocorridas nos três anos de estudo, mostram que houve um aumento gradativo e significativo nas internações psiquiátricas compulsórias no período estudado de 2009 a 2011. Estas internações representaram 0,71\% de todas as internações psiquiátricas ocorridas no mesmo período.

Tabela 1. Distribuição das Internações Psiquiátricas e Internações Psiquiátricas Compulsórias no Hospital Municipal Thelma Villova Kasprowicz, por ano, Maringá - Paraná, 2012.

\begin{tabular}{lccc}
\hline & \multicolumn{3}{c}{ ANO } \\
\cline { 2 - 4 } & $\mathbf{2 0 0 9}$ & $\mathbf{2 0 1 0}$ & $\mathbf{2 0 1 1}$ \\
\hline Internações Psiquiátricas Compulsórias & 24 & 24 & 55 \\
Internações Psiquiátricas & 4864 & 4724 & 4861 \\
\hline
\end{tabular}

Fonte: Fluxo Diário do Hospital Municipal de Maringá Thelma Villanova Kasprowicz, 2012.

Não há sazonalidade definida para as internações psiquiátricas compulsórias. No ano de 2009, ocorreram maiores registros de internação psiquiátrica compulsória no mês de março, em 2010 e 2011 houve maior registro de internação psiquiátrica compulsória no mês de julho.

A Regional de Saúde que mais efetuou registro e encaminhou como internação psiquiátrica compulsória foi a $15^{\circ}$ Regional de Saúde de Maringá com 81 registros (78,64\%). O município de Maringá foi o que mais efetuou internações compulsórias no período estudado. Isso é caracterizado pelo maior número de habitantes da 15 a Regional de Saúde e do município de Maringá e também pela proximidade ao serviço.

Também observou-se que houve em 2009 uma internação psiquiátrica compulsória pela $16^{\circ}$ Regional de Saúde, sendo uma regional de saúde fora da pactuação estabelecida pela rede estadual de saúde mental.

A Tabela 3 mostra que o agrupamento no CID10 que mais causou internações psiquiátricas compulsória nos anos pesquisados foi o F 19 (transtornos mentais e comportamentais devidos ao uso de múltiplas drogas e ao uso de outras substâncias psicoativas).

Tabela 2. Distribuição das Internações Psiquiátricas Compulsórias de acordo com o CID, por ano, 
Maringá - Paraná, 2012.

\begin{tabular}{lcccc}
\hline CID 10 & $\mathbf{2 0 0 9}$ & $\mathbf{2 0 1 0}$ & $\mathbf{2 0 1 1}$ & Total \\
\hline F 06 & 1 & 0 & 0 & 1 \\
F 06.8 & 0 & 1 & 0 & 1 \\
F 06.9 & & & & \\
F 10 & 0 & 1 & 0 & 1 \\
F 10.2 & 1 & 2 & 13 & 16 \\
F 12.2 & 3 & 1 & 2 & 6 \\
F 14 & 1 & 0 & 0 & 1 \\
F 14.2 & 1 & 0 & 0 & 1 \\
F 14.3 & 4 & 2 & 0 & 6 \\
F 19 & 0 & 0 & 1 & 1 \\
F 19.1 & 4 & 13 & 38 & 55 \\
F 19.2 & 0 & 0 & 1 & 1 \\
F 20 & 3 & 2 & 3 & 8 \\
F 31 & 4 & 2 & 1 & 7 \\
F 31.6 & 0 & 1 & 2 & 3 \\
F 60 & 0 & 1 & 1 & 2 \\
F 70 & 1 & 0 & 1 \\
F 79 & 1 & 0 & 2 & 5 \\
F 90 & 2 & 1 & 1 & 1 \\
F 91 & 0 & 1 & 1 \\
F 91.1 & 0 & 1 & 4 \\
\hline
\end{tabular}

Fonte: Fluxo Diário do Hospital Municipal de Maringá Thelma Villanova Kasprowicz, 2012.

O sexo masculino prevalece nas internações psiquiátricas compulsórias. De fato no ano de 2010 não houve nenhuma internação psiquiátrica compulsória do sexo feminino devido a instituição estar com a ala feminina em reforma. A faixa etária que prevalece nos índices de internação psiquiátrica compulsória é a de 16 a 20 anos.

Tabela 3. Distribuição da média em dias das Internações Psiquiátricas Compulsórias, por ano, no município de Maringá-Paraná, 2012.

\begin{tabular}{llll}
\hline & \multicolumn{3}{c}{ ANO } \\
\cline { 2 - 4 } & 2009 & 2010 & 2011 \\
\hline Dias de Internações Psiquiátricas Compulsórias & 88 & 39 & 109 \\
Média das Internações Psiquiátricas Compulsórias & 3,82 & 1,62 & 1,98 \\
\hline
\end{tabular}

Fonte: Fluxo Diário do Hospital Municipal de Maringá Thelma Villanova Kasprowicz, 2012.

Houve variação também na média de dias de permanência das internações ocorridas na Emergência Psiquiátrica Thelma Villanova Kasprowicz, com cerca de três dias para o ano de 2009, 
aproximadamente um dia de internação psiquiátrica compulsória em 2010 e a média aproximada de dois dias de internação psiquiátrica compulsória em 2011. Esses valores são baixos, devido ao fato destes clientes serem encaminhados a outro serviço de internação psiquiátrica hospitalar que disponibiliza tratamento com mais dias de internação psiquiátrica.

\section{DISCUSSÃO}

Quanto às três regionais de saúde referenciadas para atendimento na $E P$, podemos aferir que a regionalização dos serviços de saúde delimita-se ao objetivo de garantir acesso, resolubilidade, qualidade e integralidade aos serviços e às ações de saúde, contudo, muito além dos limites municipais, vislumbrando-se alcançar as instâncias organizadas desse tecido, tais como distritos e regiões de saúde em desenhos regionais intramunicipais ou até mesmo regiões de saúde fronteiriças (BELTRAMMI, 2008).

O CID10 que representou a maioria das internações compulsórias no período estudado foi a drogadição, termo utilizado para uso de substâncias ilícitas. O problema do uso de drogas se dá pelo encontro de um produto, de uma personalidade e de um momento sócio-cultural, ou seja, a droga não é mais simplesmente um produto, mas um fenômeno (PEREIRA; SUDBRACK, 2008).

É muito provável que este fato seja reflexo do surto epidêmico de uso que vem grassando em nosso meio desde os meados dos anos 80 . Tem sido admitido, pela incidência crescente e pela relevância dos problemas a ela relacionados. Esta ascensão tem trazido problemas para os serviços públicos, desde o sistema de justiça criminal até os serviços sociais e de saúde, demandando remodelações conceituais e estruturais destes, incluindo a interação em suas ações (BORINI; GUIMARÃES; BORINI, 2003).

As internações compulsórias apresentaram comorbidades que pode ser definidas como a ocorrência de duas entidades diagnósticas em um mesmo indivíduo. O abuso de substâncias é o transtorno coexistente mais frequente entre pessoas com transtornos mentais. Entre os transtornos mais comuns com estreita correlação com o abuso de substâncias incluem-se os transtornos de humor, como a depressão, transtornos de ansiedade, transtornos de conduta, déficit de atenção e hiperatividade e, numa extensão menor, a esquizofrenia, transtornos alimentares e transtornos da personalidade (ZALESKI, 2006).

As maiores dificuldades na abordagem da pessoa com comorbidade está no diagnóstico, pois ocorre uma "superposição de sintomas". Um transtorno pode exacerbar ou mascarar o outro. É difícil estabelecer diferenças entre a presença de comorbidade psiquiátrica e abuso de 
substâncias psicoativas (ZALESKI, 2006).

A quase totalidade das internações psiquiátricas compulsórias encontradas nesse estudo foi de jovens do sexo masculino. Estudos mostram que as mulheres apresentam maiores taxas de prevalência de transtornos de ansiedade e do humor que homens, enquanto estes apresentam maior prevalência de transtornos associados ao uso de substâncias psicoativas, incluindo álcool, transtornos de personalidade anti-social e esquizotípica, transtornos do controle de impulsos e de déficit de atenção e hiperatividade na infância e na vida adulta (ANDRADE; VIANA; SILVEIRA, 2006).

\section{CONCLUSÃO}

A lei da reforma psiquiátrica tem sido reconhecida no âmbito da saúde mental, proporcionando melhores formas de tratamento para as pessoas com transtorno mental, hoje a internação psiquiátrica compulsória é uma modalidade que é pouco utilizada devido a burocracia jurídica, mas tem sido muito utilizada no âmbito de internações psiquiátricas por drogadição nos adolescentes.

De forma assertiva esse estudo corrobora que os adolescentes são os responsáveis pela maioria das internações psiquiátricas compulsórias por drogadição, as políticas públicas podem ser transformadas em ações efetivas e resolutivas, com serviços substitutivos e profissionais habilitados para atuarem com este público alvo.

Quando falamos da reforma psiquiátrica logo vem a questão da "inserção social”, mas não basta inserirmos estas pessoas, temos que acompanhar este processo, é um trabalho que envolve muitos profissionais.

A internação psiquiátrica compulsória, quando bem aplicada, é um meio de assegurar o direito a saúde, pois as pessoas que utilizam este serviço são pessoas que precisam de uma intervenção nas ações de saúde que visem melhoria da qualidade de vida. Mas, ao mesmo tempo em que tem esse papel, a internação psiquiátrica compulsória acaba destituindo a liberdade de a pessoa escolher o tratamento ao qual quer ser tratado, e a liberdade de ir ou vir é subtraída.

\section{REFERÊNCIAS}

Andrade LHSG, Viana MC, Silveira CM. Epidemiologia dos transtornos psiquiátricos na mulher. Revista de psiquiatria clínica [online]. 2006; 33(2):43-54.

Beltrammi DGM. Descentralização: o desafio da regionalização para Estados e Municípios. RAS . 2008;10(41). 
Borini P, Guimarães RC, Borini SB. Usuários de drogas ilícitas internados em hospital psiquiátrico: padrões de uso e aspectos demográficos e epidemiológicos. Jornal brasileiro de psiquiatria. 2003; $52(3)$.

Britto RC. A internação psiquiátrica involuntária e a Lei 10.216/01. Reflexões acerca da garantia de proteção aos direitos da pessoa com transtorno mental. Rio de Janeiro: s.n., 2004. 210p.

Dias CB, Silva AL. O perfil e a ação profissional da (o) enfermeira (o) no Centro de Atenção Psicossocial. Revista escola de enfermagem USP [online]. 2010; 44(2):469-475. http://dx.doi.org/10.1590/S0080-62342010000200032

Fortes HM. Tratamento compulsório e internações psiquiátricas. Revista brasileira de saúde materno infantil [online]. 2010; 10(2):s321-s330.

Marques ACPR, Cruz MS. O adolescente e o uso de drogas. Revista brasileira de psiquiatria. [online]. 2000; 22(2):s32-s36. http://dx.doi.org/10.1590/S1516-44462000000600009

Mendes ACG, Silva Junior JB, Medeiros KR, Lyza TM, Melo Filho DA, Sá DA. Avaliação do Sistema de Informações HospitalaresSIH/SUS como Fonte Complementar na Vigilância e Monitoramento de Doenças de Notificação Compulsória. 2000; 9(2).

Mielke FB, Kantorski LP, Olschowsky A, Jardim VMR. Características do cuidado em saúde mental em um CAPS na perspectiva dos profissionais. Revista trabalho, educação e saúde [online]. 2011; 9(2):265-276. http://dx.doi.org/10.1590/S1981-77462011000200006

Nagaoka AP, Furegato ARF, Santos JLF. Usuários de um centro de atenção psicossocial e sua vivência com a doença mental. Revista escola de enfermagem USP [online]. 2011; 45(4): 912-917. http://dx.doi.org/10.1590/S0080-62342011000400017

Pereira SEFN, Sudbrack MFO. Drogadição e Atos Infracionais na Voz do Adolescente em Conflito com a Lei. Psicologia: Teoria e Pesquisa 2008; 24(2):151-159. http://dx.doi.org/10.1590/S0102$\underline{37722008000200004}$

Zaleski M, Laranjeira RR, Marques ACPR, Ratto L, Romano M, Alves HNP, Soares, MBM, Abelardino V, Kessler F, Brasiliano S, Nicastri S, Hochgraf PB, Gigliotti AP, Lemos, T. Diretrizes da Associação Brasileira de Estudos do Álcool e outras Drogas (ABEAD) para o diagnóstico e tratamento de comorbidades psiquiátricas e dependência de álcool e outras substâncias. Revista brasileira de psiquiatria [online]. 2006; 28(2):142-148. http://dx.doi.org/10.1590/S1516-44462006000200013 